\title{
Engagement, redes sociales y moda internacional. La boda real de Harry-Meghan Markle
}

\author{
Engagement, social networks and international fashion. Harry- \\ Meghan Markle's wedding with
}

\author{
Villena Alarcón, E. y Segarra-Saavedra, J. ${ }^{1}$ \\ Recibido: 11-03-2020 - Aceptado: 15-08-2020 \\ https://doi.org/10.26441/RC19.2-2020-A17
}

\begin{abstract}
RESUMEN: La evolución de Internet y la penetración de las redes sociales ha modificado la forma de comunicar que tenía la moda. Atrás han quedado modelos de comunicación que situaban a los medios tradicionales en el centro de su gestión estratégica. Las nuevas tendencias contextualizan las redes sociales como eje vertebrador de la relación con los públicos. Las bodas reales son una herramienta fundamental para las firmas de moda participantes en el evento gracias a su amplio potencial como instrumento para difundir mensajes. El texto apuesta por el estudio de caso múltiple para vincular las principales marcas de moda internacionales con los eventos especiales y acude al análisis de contenido de los perfiles de redes sociales en los que las firmas protagonistas tienen presencia. Los resultados revelan cómo los públicos reaccionan a los esfuerzos comunicativos realizados y lo pertinente de recurrir a estos actos para mejorar el compromiso con los stakeholders.
\end{abstract}

Palabras clave: engagement; formato publicitario no convencional; stakeholders; moda; relaciones públicas; boda real.

\begin{abstract}
The speedy evolution of Internet and the penetration of social networks has changed the way of communicating that was usually had the fashion. Gone are the models of communication that placed traditional media at the centre of their strategic management. The new trends contextualize social networks as the backbone of the relationship with audiences. In this sense, real weddings have become a fundamental tool for the fashion brands participating in the event thanks to their wide potential as an instrument to spread the messages. The text opts for multiple case studies to link the main international fashion brands with special events and goes to the content analysis of the profiles of social networks in which the leading firms have a presence. The results reveal how audiences react to the communication efforts made and the pertinence of resorting to these acts to improve engagement with stakeholders.
\end{abstract}

Keywords: engagement; unconventional ad format; stakeholders; fashion; public relations; royal wedding

\footnotetext{
${ }^{1}$ Eduardo Villena Alarcón es Doctor en Comunicación Organizacional y Profesor de Creación de campañas en entornos digitales y Dirección estratégica en publicidad del Departamento de Comunicación Audiovisual y Publicidad de la Universidad de Málaga. eduardo.villena@uma.es http://orcid.org/0000-0001-8798-8506

Jesús Segarra-Saavedra es Doctor en Comunicación y Profesor de Gestión de la información en Comunicación e Innovación en la comunicación de las industrias creativas del Departamento de Comunicación y Psicología Social de la Universidad de Alicante. jesus.segarra@ua.es http://orcid.org/0000-0001-9420-5258
} 


\section{Introducción}

La comunicación de las empresas con sus públicos ha sufrido cambios importantes y muy relevantes en el último cuarto de siglo. Esto no es sólo producto del surgimiento de Internet, sino también de la aparición y el uso extendido de las redes sociales, que cuentan actualmente con 3.484 millones de usuarios activos en todo el mundo, lo que supone una penetración del 45\% (VV.AA., 2019). Este hecho ha convertido a las redes sociales en una fuente de datos sobre los públicos a los que las empresas dedican su actividad (Laurell \& Sandstrom, 2013; Lin, 2018). Del mismo modo, a través del amplio desarrollo de las herramientas de monitorización, cualquier organización puede conocer de manera gratuita en tiempo real y a nivel global cuáles son las preferencias de sus stakeholders, así como saber desde dónde se conectan o cuál es el sentimiento hacia la marca (Cvijikj \& Michahelles, 2013; Arora, Bansal, Kandpal, Aswani \& Dwivedi, 2019). Por tanto, los social media responden a un instrumento relevante de relaciones públicas (Phan, 2011) que las empresas deben introducir si quieren transmitir de manera eficiente sus mensajes y conocer las reacciones generadas por el usuario. Por otro lado, la gestión de la comunicación con los públicos resulta un tema de interés para las empresas y los academicistas. Investigaciones previas se han centrado en cómo las redes sociales han generado oportunidades de comunicación y han favorecido la transmisión de mensajes relacionados con la organización (Diga \& Kelleher, 2009; Verhoeven, Tench, Zerfass, Moreno \& Vercic, 2012).

A pesar de que este canal ha ayudado a personalizar la comunicación (Mangold \& Faulds, 2009) con los públicos, la multidireccionalidad del mensaje en redes sociales ha obligado a los profesionales del sector a identificar las necesidades de las personas para conectar con ellas y que éstas produzcan y compartan contenido online (Boyd \& Ellison, 2008). A través de los comentarios de los usuarios o mediante la observación de las reacciones en las redes sociales, una empresa puede conocer los intereses de los usuarios, lo que favorece la participación de los miembros en la creación de contenido (Palmer \& Koenig-Lewis, 2009).

En un mundo tan voluble y cambiante como el sector textil, desde el punto de vista de las relaciones públicas, conocer a tiempo real cuáles son los intereses de los públicos es una oportunidad para quienes aspiran a generar contenido de calidad, feedback y aumento de ventas. En el ámbito de la moda, distintas empresas comienzan ya a estudiar las características de sus públicos y sus reacciones a las informaciones compartidas. Dentro de este particular, las bodas reales son uno de los eventos especiales más importantes para los relacionadores públicos especializados en moda, las cuales no solo se circunscriben a la esfera offline sino que su actividad se refleja en el ámbito online. Aquí las redes sociales se han institucionalizado como una herramienta de amplificación de los mensajes que ha facilitado la participación de actores previamente excluidos (McQuarrie, Miller \& Phillips, 2013) y ha acercado la firma al consumidor final (Mohr, 2013). En función de los resultados obtenidos y de la literatura existente, el texto contribuye al campo de las relaciones públicas y la gestión de redes sociales en la comunicación de la moda vinculada a eventos especiales con la producción de mensajes y el compromiso que esto genera. Del mismo modo, contribuye a entender cómo las empresas de moda estructuran su comunicación en torno a una Boda Real.

La investigación -que al igual que lo aportado por Hon \& Grunig (1999), recoge la reacción de los públicos- resulta pertinente porque no existen estudios previos que examinen la respuesta online de los usuarios a los mensajes emitidos por las empresas de moda en redes sociales. A pesar de la oportunidad que supone conocer cómo responden los públicos a los esfuerzos comunicativos, aún no existen investigaciones similares a partir de diferentes perspectivas (Richter, Riemer \& Vom, 2011) debido a la dificultad que entraña la recogida de datos. Por tanto, el estudio pretende ofrecer información sobre cómo se construyen las relaciones y permite analizar la efectividad de las comunicaciones con la intención de reducir la brecha entre los social media y las relaciones públicas. 


\section{Marco referencial}

Diferentes investigaciones tienen como objeto de estudio las relaciones entre las empresas y sus públicos a través del ámbito online (Diga \& Kelleher, 2009; Carr \& Hayes, 2015) debido a la capacidad de interacción que presentan. En términos generales, estos análisis se han preocupado de contabilizar los esfuerzos que las empresas realizan; sin embargo, son exiguos las investigaciones que se ocupan de cómo los públicos reciben los mensajes que las organizaciones comparten (Saxton \& Waters, 2014) en las redes sociales de manera inmediata. Facebook, Twitter y más recientemente Instagram han supuesto un revulsivo para el campo de las relaciones públicas (Fernández Souto, 2018) debido, no solo a la oportunidad de producir diálogo, sino por la oportunidad que presenta de conocer de manera instantánea la reacción a los mensajes.

Las redes sociales responden ya como un canal de comunicación al servicio de las organizaciones y han sido incorporadas a pleno rendimiento al plan de relaciones públicas (Curtis, Edwards, Frasier, Gudelsky, Holmquist, Thornton \& Sweetser, 2010) como un instrumento más a la hora de difundir contenido y generar conversación. No obstante, como elemento estratégico (Breakenridge, 2012), su impacto debe ser medido para conocer el grado de compromiso adquirido por los públicos a través de los likes, comentarios y compartidos (Cvijikj \& Michahelles, 2013). Las relaciones públicas recurren a las redes sociales como medio para amplificar sus mensajes y generar diálogo (Waters \& Jamal, 2011), sin embargo, esto obliga a conocer cómo esa información está impactando en los públicos para lograr una comunicación eficaz y que ésta revierta positivamente en la empresa.

Las distintas redes sociales han sido objeto de estudio en diferentes investigaciones relacionadas con los públicos (Ruehl \& Ingenhoff, 2015; Dhanesh, 2017). Reveladoras son aquellas que se han ocupado de conocer la estrategia de comunicación en Facebook de las ONG (Waters, Burnett, Lamm \& Lucas, 2009; Saxton \& Waters, 2014) o las empresas cotizadas (Kim, Kim \& Sung, 2014). Esto encuentra su reflejo en Twitter, cuyos estudios se centran en definir la presencia de las no gubernamentales (Waters \& Jamal, 2011; Himelboim, Golan, Moon \& Suto, 2014) o las empresas presentes en Fortune 500 (Rybalko \& Seltzer, 2010). Finalmente, la revisión de la literatura revela a Instagram como una genuina herramienta de relaciones públicas (Wright \& Hinson, 2013; Guidry, Messner, Jin \& Medina-Messner, 2015; Guidry, Jin, Orr, Messner \& Meganck, 2017).

\subsection{Eventos especiales y relaciones públicas en redes sociales}

Las bodas reales se han convertido en nuevos espacios de relaciones públicas para el ámbito de la moda. El enlace del Príncipe Enrique, Duque de Sussex, con Meghan Markle, que tuvo lugar el 19 de mayo de 2018 en Clarence House, se desarrolló como un acontecimiento especial que las marcas de moda utilizaron para difundir sus mensajes a través de los diferentes canales. En base a esto, se reprodujo el concepto tradicional definido por Grunig \& Hunt (1984) en tanto en cuanto se direccionó y se gestionó la comunicación entre una organización y sus públicos. No obstante, mucho han evolucionado los enlaces reales desde que Isabel II contrajera matrimonio con el Duque de Edimburgo en 1947, primera Boda Real televisada en directo. Con el devenir de los años, estos eventos han ganado en participación y difusión debido al amplio desarrollo de Internet y la evolución de las redes sociales, las cuales han convertido estos actos en un evento donde los actores participantes pueden transmitir contenido al resto de públicos. Se cumple aquí lo postulado por Almansa y Navarrete (2012) cuando afirman que el paradigma social actual ha basculado hacia un proceso en el que son los propios usuarios los encargados de construir la información y difundirla.

El ceremonial presente en este acto público oficial responde a un enfoque que, desde la perspectiva de la comunicación, plantea nuevas relaciones, ya que cuenta una historia y crea un ámbito propio de reconocimiento en el que confluir (Otero, 2004). Sin embargo, esto resultaría un enfoque re- 
duccionista si se obviaran a todos los demás sujetos. Al intento de proyectar una imagen sólida y positiva de la Institución, hay que añadirle los objetivos de los otros participantes. A este respecto, la propia Otero (2000) ya expuso que el gran reto del protocolo en la actualidad era incorporar los agentes sociales y hacerlos visibles; algo que no ha sido posible hasta que las redes sociales se han generalizado y han comenzado a ser parte de la comunicación institucional.

En base a esto, resulta fundamental conocer cómo las empresas de moda conformar constelaciones (Chaplin \& Lowrey, 2010) en torno a un acto público; es decir, cómo se relacionan a través de redes sociales a tenor de una ceremonia mediática. Dado el desarrollo y la integración cada vez mayor de las empresas de moda en los entornos digitales (Laurell, 2014), es necesario saber la forma en la que se articulan las relaciones en este medio en el contexto de las bodas reales y cuáles son las reacciones de los públicos. Tal y como se expone previamente, este campo de conocimiento no ha sido estudiado, aunque la moda sí ha sido analizada tanto desde la perspectiva de la publicidad (Pires, 2014), la sociología (Oliveira, 2013; Machuco, 2013; Cereda, 2014; Gama, 2014) o la teoría de la moda (Cucinotta, 2017).

A pesar de que las ceremonias religiosas han sido objeto de estudio desde las relaciones públicas (Ramos Fernández, 2010) y que distintos autores se han ocupado de conocer la historia del ceremonial (Sánchez, Gómez y Pérez, 2015) y de definir los actos oficiales desde diferentes ámbitos del conocimiento (Otero, 2000; Fuente, 2010; Barquero y Castillo, 2011; Sánchez, 2011; Fernández, 2004), así como la importancia de los públicos (Castillero, 2014); son escasas las aportaciones que relacionan las bodas reales con los actores implicados por lo que la pertinencia de la investigación se encuentra avalada por la necesidad que tienen las firmas de moda de conocer cómo se implementan los mensajes en redes sociales para poder establecer estrategias solventes adaptadas a los objetivos planteados por las firmas para, de esta manera, alcanzar las metas fijadas a la hora de participar en eventos especiales.

\section{Metodología}

La presente investigación aboga por un estudio empírico de carácter cuantitativo que vincule las principales marcas de moda y los actos públicos. Específicamente, el texto persigue identificar cómo se relacionan las empresas internacionales del sector textil con sus públicos en torno a un evento especial como es la Boda Real del Príncipe Enrique de Sussex con Meghan Markle. Para ello, se presenta una investigación exploratoria (Lofman, 1991; Park, Song \& Ko, 2011; Nash, 2019) que permite conocer cuál ha sido el esfuerzo comunicativo que han realizado las firmas a tenor del enlace y el compromiso adquirido por parte de los públicos.

Teniendo en cuenta lo anterior, el objetivo principal de la investigación estriba en conocer si la presencia de marcas de moda en una Boda Real logra, en términos de comunicación, un compromiso social mayor que la que habitualmente alcanza con su actividad cotidiana en sus redes sociales. El logro de este objetivo posibilita a posteriori la aplicación práctica en la toma de decisiones profesionales, dado que aporta información de interés para las firmas que se encuentren interesadas en participar de forma activa en este tipo de actos equipando a los asistentes. Del mismo modo, para examinar un fenómeno social tan complejo dentro de la moda, se acude al estudio de caso múltiple a partir de diferentes datos que enriquecen los resultados (Miles \& Huberman, 1994). Con ello se asegura una comprensión holística de los eventos (Yin, 1994) y entender los matices entre el hecho y su contexto (Aaboen, Dubois \& Lind, 2012). Ahondando en ello, y a pesar de que el texto no plantea ninguna hipótesis debido a que responde a un tema estudiado en anterioridad de manera exigua (Hernández, Fernández y Baptista, 2007), sí parte de diferentes preguntas de investigación: 
- PI1: ¿Cuál es el esfuerzo comunicativo en redes sociales de las marcas que participan en los eventos especiales?

- PI2: ¿Cómo responden los públicos al esfuerzo comunicativo que hacen las marcas de moda ante un evento especial?

Para intentar responder a las anteriores cuestiones se realizó un muestreo no probabilístico (Vieira, 2009) de sujetos-tipo entre todas las firmas que confluyeron en dicho acto. Particularmente, se optó por un muestreo no probabilístico deliberado ante la dificultad de acceder a la población completa en lo que respecta al marco muestral; por lo que las unidades que componen la muestra responden a los criterios determinados a partir del diseño de la investigación; es decir, que fueran firmas de moda, que generaran engagement y que publicaran contenido habitualmente en sus perfiles. Además, las marcas que componen el corpus de estudio (Ver Tabla 1) debían cumplir como requisito sine qua non con las siguientes especificaciones:

- Que tuviera presencia en alguna de las tres redes sociales objeto de estudio.

- Que hubiera vestido a alguno de los sujetos implicados en el evento.

- Que hubiera generado contenido en torno al evento.

En última instancia, y tras detectar ciertas limitaciones, se procedió a una observación previa de todas las firmas participantes que fueron objeto de publicación en los principales diarios británicos para asegurar la pertinencia de la muestra, las empresas propias del ámbito que conformaron el corpus de estudio a fecha de codificación fueron:

Tabla 1. Corpus de estudio

\begin{tabular}{|l|r|r|r|}
\multicolumn{1}{|c|}{ Marca } & Facebook & \multicolumn{1}{c|}{ Twitter } & \multicolumn{1}{c|}{ Instagram } \\
\hline Givenchy & 2.751 .468 & 976.000 & 12.610 .000 \\
\hline Stella McCarney & 919.577 & 1.030 .000 & 5.816 .700 \\
\hline Óscar de la Renta & 1.153 .308 & 437.000 & 3.796 .800 \\
\hline Stewart Parvin & 7.088 & 4.786 & 6.379 \\
\hline Dior & 16.395 .717 & 8.043 .000 & 24.515 .130 \\
\hline Dolce \& Gabanna & 11.513 .263 & 5.253 .000 & 20.174 .394 \\
\hline Versace & 5.291 .851 & 4.634 .000 & 17.224 .686 \\
\hline Valentino & 2.875 .427 & 2.041 .000 & 12.165 .500 \\
\hline The Fold & 31.688 & 3.359 & 22.070 \\
\hline Victoria Beckham & 3.138 .427 & 12.780 .000 & 24.850 .000 \\
\hline
\end{tabular}

Fuente: Elaboración propia a partir del número de seguidores

Tras seleccionar la muestra de estudio, se procedió a aplicar la técnica de investigación elegida, el análisis de contenido (Alonso-González, 2015; García, Miquel-Segarra \& Navarro-Beltrá, 2018; Pérez \& Sanz, 2019), a cada uno de los perfiles donde tenían presencia para conocer el esfuerzo comunicativo e identificar el comportamiento de los públicos en términos de me gusta, comentarios y compartidos. Esto permitió conocer cómo las empresas participantes implementaron las redes sociales para transmitir sus mensajes a tenor de la Boda Real y cómo lo recibe la audiencia social. La pertinencia de la técnica viene avalada por lo aportado por Piñuel (2002) cuando postula que el análisis de contenido permite procesar datos de manera cuantitativa a partir de un proceso comunicativo registrado previamente. Para cada una de las empresas se ha contabilizado el contenido que las firmas volcaron en Facebook, Twitter e Instagram, al ser estas las herramientas más útiles a la hora de difundir sus informaciones a los públicos. Así lo atestigua el último informe remitido por USC 
Annenberg Center for Public Relations (2019) al situar al terceto social entre las plataformas con mayor efectividad a la hora de mejorar las estrategias de comunicación. Los profesionales de las relaciones públicas consideran Instagram (46\%) como el social media más importante dentro de su planificación, seguido de Twitter (31\%) y Facebook (30\%). Desde la perspectiva de los internautas, $\mathrm{y}$ en función de los datos aportados por el último informe We are social (Kemp, 2019), los usuarios activos en redes sociales ascienden en 2019 a 3.484 millones de personas, lo que supone una penetración del $45 \%$. El reporte sitúa las redes sociales analizadas entre las más visitadas. Ante este particular, Facebook, tercera en el listado, registra un total de 2,271 usuarios, Twitter, séptima, 250,8 millones e Instagram, décima, 894,9 millones de personas.

- PI3: ¿Resulta eficiente para las marcas de moda publicar contenido en esos eventos?

Para encontrar respuesta a la tercera pregunta se apostó por un enfoque longitudinal que permitiera conocer cómo las diferentes variables y sus relaciones evolucionan a través del tiempo (Hernández, Fernández y Baptista, 2007) en diferentes momentos. Se comparó pues (a) las reacciones generadas por las publicaciones sobre la Boda Real con aquellas cosechadas por (b) las actualizaciones habituales que más engagement habían generado a lo largo del tiempo. Con ello, se dilucida si el esfuerzo comunicativo llevado a cabo en el evento especial resulta conveniente y, por ende, si su participación en dicho evento resulta rentable en términos de comunicación. Cabe destacar que, en términos de Cvijikj \& Michahelles (2013), la presente investigación repara en las "reacciones", entendiendo estas como el compromiso adquirido por los públicos manifestado a través de los likes, comentarios y compartidos. Estas variables resultan fundamentales a la hora de estudiar la actividad de las marcas en medios sociales.

En suma, la investigación se centra en dos elementos básicos: (1) conocer si el contenido propio de un evento especial publicado por una firma de moda desencadena un mayor nivel de participación que el contenido habitual y (2) dónde es compartido. A pesar de que la Boda Real se produjo el 19 de mayo de 2018 a las 13:00 hora local, la investigación aplicada cubre los días 19 y 20, debido a que diferentes firmas se encuentran localizadas en EE. UU y existe diferencia horaria. Del mismo modo, se amplifica la recogida de datos hasta el 26 de mayo de 2018 debido a que se detectó en una observación previa que ciertas empresas habían dilatado las actualizaciones a lo largo de los días posteriores. Las bases de datos del análisis utilizadas responden a aspectos comunes en los estudios de redes sociales (Guo \& Saxton, 2014; Sullivan, Schneiders, Cheang, Kitto, Lee, Redhead, Ward, Ahmed \& McCrory, 2012) como el número de actualizaciones o las reacciones obtenidas. La medición de estas reacciones de los públicos a los mensajes permitió analizar el compromiso, sentimiento e importancia a través del conteo de:

- La cantidad de comentarios, que indica el compromiso de la empresa.

- La cantidad de "me gusta", que indica el sentimiento del público, además del número aproximado de lectura.

- La cantidad de acciones compartidas, que indica disposición del usuario de convertirse en embajador a la hora de difundir el mensaje.

Asimismo, para descargar los posts de todas las redes sociales se implementó un código propio de Python que permitió contabilizar el número de reacciones. Este lenguaje de programación que automatiza la recuperación de las reacciones ya ha sido aplicado con éxito en diferentes investigaciones relacionadas con la comunicación online (Debatin, Lovejoy, Horn \& Hughes, 2009; Lovejoy \& Saxton, 2012; Saxton \& Waters, 2014). Del mismo modo, tras recopilar los datos, se exportaron al paquete de software IBM SPSS Statistics (versión 22) donde se combinaron diferentes tipos de análisis (Esteban-Santos, García, Carey \& Bellido-Pérez, 2018). Los estudios de frecuencias, los 
promedios y otras medidas de correlación se utilizaron para describir las relaciones entre las diferentes empresas que conforman el corpus.

\section{Análisis de los resultados}

A continuación los resultados se agrupan de la siguiente manera. En primer lugar, un epígrafe con los datos del engagement alcanzado por parte de las firmas de moda con las actualizaciones realizadas en torno a la Boda Real. Asimismo, un segundo epígrafe recoge. las reacciones a las publicaciones habituales que han generado más engagement a lo largo del tiempo en las cuentas objeto de estudio.

\subsection{Reacciones generadas por las publicaciones sobre la Boda Real}

Una vez ejecutada la investigación aplicada, las empresas objeto de estudio presentaban en Facebook un promedio de seguidores de 4.407.781,00 que varían desde un mínimo de 7.088,00 a un máximo de 16.395.717,00. Dado que la primera pregunta de investigación buscaba conocer los esfuerzos comunicativos que realizaron las empresas durante el evento, resulta necesario examinar cómo se comportaron ante el hecho. El promedio de estados publicados aquí fue de 9, con un rango de 0 a 9. En función de lo anterior, la firma que más publicó fue Givenchy, responsable de uno de los vestidos de novia, mientras que Victoria Beckham no realizó actualizaciones en ninguna red social, a pesar de haber vestido a distintos invitados. Particularmente, la red social más utilizada fue Instagram. Dior, Óscar de la Renta, The Fold y Valentino se decantaron por no publicar en Facebook. De cualquier forma, esta investigación no está centrada exclusivamente en las organizaciones sino en los públicos. De hecho, el interés radica también en las reacciones de los usuarios al contenido generado por las firmas. Para dar respuesta a la segunda pregunta, la Tabla 2 muestra el promedio de likes $(6.925,00)$ respecto al total $(69.250,00)$. Específicamente, el análisis indica que la empresa que más "me gusta" aglutina es Stella McCarney, con un máximo de 33.900,00. Del mismo modo, se observa el promedio de contenido comentado $(242,50)$ y compartido $(2197,50)$.

Tabla 2. Estadístico descriptivo de las variables en Facebook

\begin{tabular}{|l|l|l|l|l|}
\hline \multirow{2}{*}{$\mathbf{N}$} & \multicolumn{1}{|c|}{ Válido } & 10 & 10 & 10 \\
\cline { 2 - 5 } & Perdidos & 0 & 0 & 0 \\
\hline Media & 6925,00 & 242,50 & 2197,50 \\
\hline Mediana & 162,50 & 16,00 & 30,00 \\
\hline Desviación & 11615,650 & 442,744 & 4865,285 \\
\hline Rango & 33900 & 1356 & 15000 \\
\hline Mínimo & 0 & 0 & 0 \\
\hline Máximo & 33900 & 1356 & 15000 \\
\hline Total & 68250 & 2425 & 21975 \\
\hline
\end{tabular}

Fuente: Elaboración propia

De manera específica, el post más compartido de Stella McCartney responde al segundo vestido de novia (1,7 mil). Del mismo modo, y tal y como se puede observar en la Imagen 1, la actualización que más reacciones generó $(21$ mil) fue la realizada por Dolce and Gabbana. Fue también la que más veces se comentó (651). 
Imagen 1. Actualizaciones en Facebook
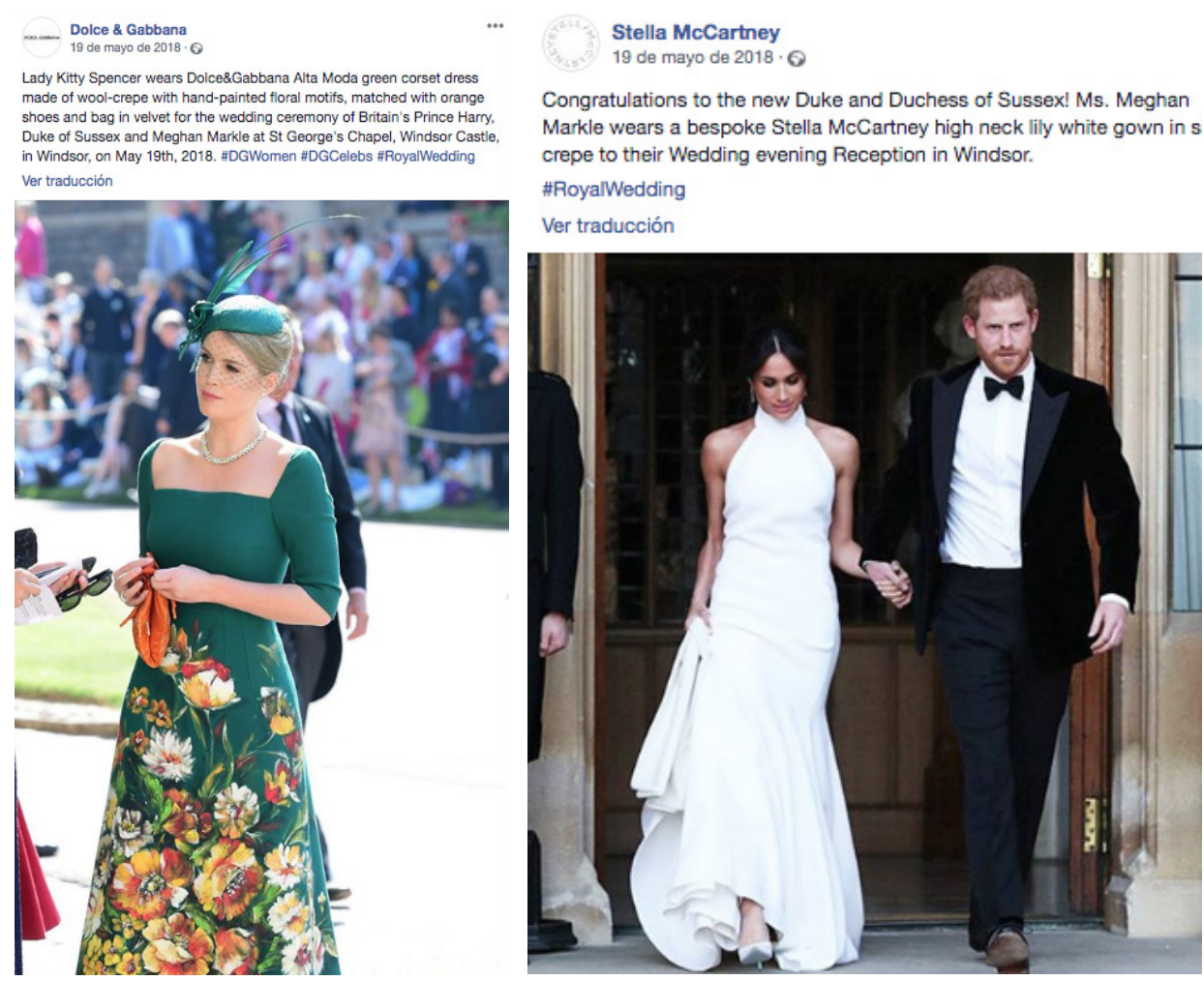

Fuente: www.facebook.es

El total de seguidores asciende en Twitter a 6.400.390,00 con un máximo de 35.202.145,00 y un mínimo de 3.359,00. Fue Stella McCartney quien más recurrió a esta red social. Presenta 14 estados publicados con un rango de 5 a 0 . La respuesta de los públicos se puede comprobar en la Tabla 3. Aquí se registran 3.493,40 likes, amén de 919,50 retuits y 63,10 comentarios de media. Se encuentran 33.900,00 de likes, 1.356,00 de comentarios y 15.000,00 compartidos de máximo. En este particular fue Givenchy quien más likes $(1.5700,00)$ y retuits $(5.269,00)$ alcanza. No obstante, Stella McCartney es quién más conversación $(2.515,00)$ genera.

Tabla 3. Estadístico descriptivo de las variables en Twitter

\begin{tabular}{|l|l|l|l|l|}
\hline \multicolumn{2}{|c|}{} & \multicolumn{1}{|c|}{ \#Likes } & \multicolumn{1}{c|}{ \# Comentarios } & \multicolumn{1}{c|}{ \# RT } \\
\hline \multirow{2}{*}{ N } & Válido & 10 & 10 & 10 \\
\cline { 2 - 5 } & Perdidos & 0 & 0 & 0 \\
\hline Media & 3493,40 & 63,10 & 919,50 \\
\hline Mediana & 99,50 & 6,00 & 22,50 \\
\hline Desviación & 5836,953 & 110,856 & 1721,468 \\
\hline Rango & 15700 & 327 & 5269 \\
\hline Mínimo & 0 & 0 & 0 \\
\hline Máximo & 15700 & 327 & 5269 \\
\hline Total & 34934 & 631 & 9195 \\
\hline
\end{tabular}

Fuente: Elaboración propia

Completando lo anterior, la actualización realizada por Givenchy, responsable del primer vestido, y que tuvo como objeto a Meghan Markle entrando en San Jorge, fue la más retuiteada (4,3k) y la que más likes generó (12k). Sin embargo, el mayor número de comentarios lo generó de nuevo Stella McCartney (166) con este tuit (Ver Imagen 2). 
Imagen 2. Actualizaciones en Twitter

Givenchy @ @ivenchy · 19 may. 2018 마

Ms. Meghan Markle, wearing a \#Givenchy Haute Couture wedding gown designed by \#ClareWaightKeller to the \#RoyalWedding

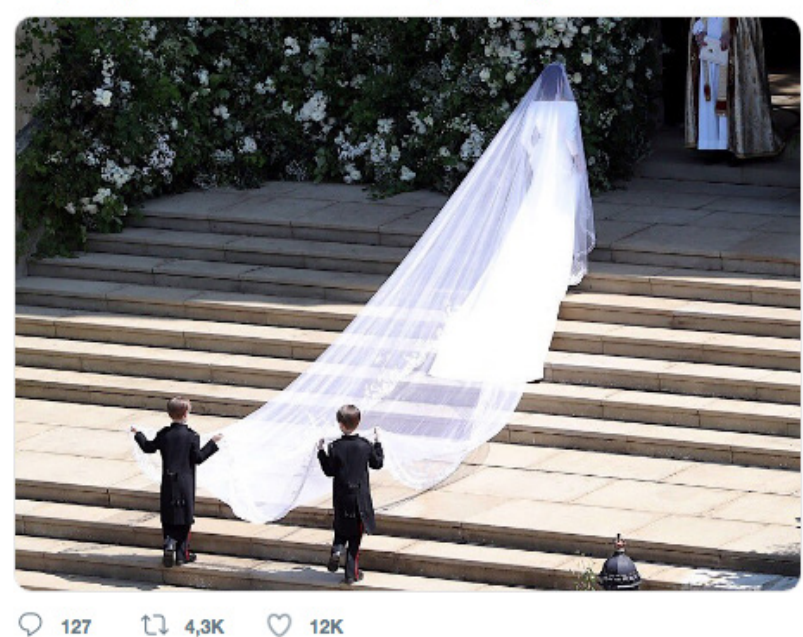

Stella McCartney @ @StellaMcCartney · 19 may. 2018 a Ms. Meghan Markle wears a bespoke Stella McCartney high neck lily white gown in slik crepe to her Wedding evening Reception with Prince Hary i

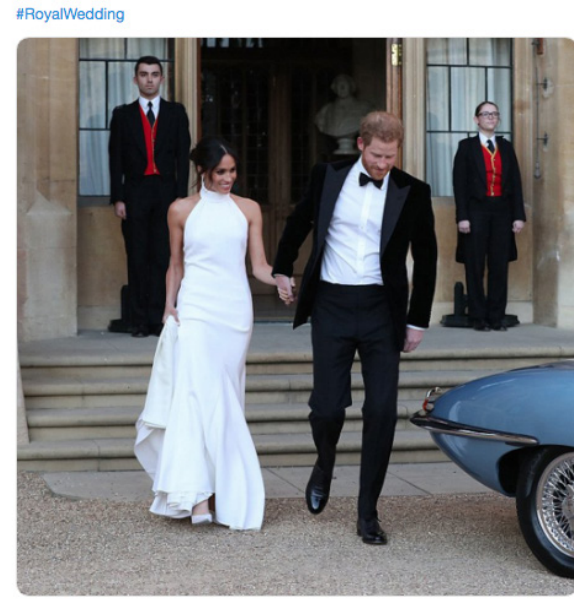

Q $166 \quad$ [T $1,4 \mathrm{~K} \quad \mathrm{O}_{7,1 \mathrm{~K}}$

Fuente: www.twitter.com

Por otra parte, las firmas que conforman el corpus el estudio aglutinan una media de 22.033.029,00 seguidores en Instagram, con máximo de 121.181.659,00 y mínimo de 6.379. Aquí se realizan 28 publicaciones con un rango de 9 a 0 . Givenchy es la empresa que más publica y la que más likes $(3.371 .000,00)$ y comentarios $(16.697,00)$ logra; muy por encima de la media de likes $(8.892 .079,00)$ $\mathrm{y}$ comentarios $(4.355,00)$.

Tabla 4. Estadístico descriptivo de las variables en Instagram

\begin{tabular}{|l|l|l|l|}
\multicolumn{2}{|c|}{} & \multicolumn{1}{|c|}{ \#Likes } & \#Comentarios \\
\hline \multirow{2}{*}{$N \quad$ Válido } & 10 & 10 \\
\cline { 2 - 4 } \multicolumn{1}{|c|}{ Perdidos } & 0 & 0 \\
\hline Media & 889207,90 & 4355,00 \\
\hline Mediana & 224000,00 & 1072,00 \\
\hline Desviación & 1297680,697 & 6196,727 \\
\hline Rango & 3371000 & 16697 \\
\hline Mínimo & 0 & 0 \\
\hline Máximo & 3371000 & 16697 \\
\hline Total & 8.892 .079 & 43.550 \\
\hline
\end{tabular}

Fuente: Elaboración propia

A razón del total de likes $(8.892 .079,00)$ se detecta un máximo de 3.371.000,00 con una desviación de 1.297.680,697 y una mediana de 224.000,00. Frente a los 43.550,00 comentarios del total, un máximo de 16.697,00.

La presentadora Oprah protagonizó el post con más reproducciones $(1.2 \mathrm{~m})$ en Instagram a través de la cuenta de Stella McCartney; la cual también fue la firma que más comentarios (7.723) generó con un carrusel sobre los Duques. 
Imagen 3. Actualizaciones en Instagram.
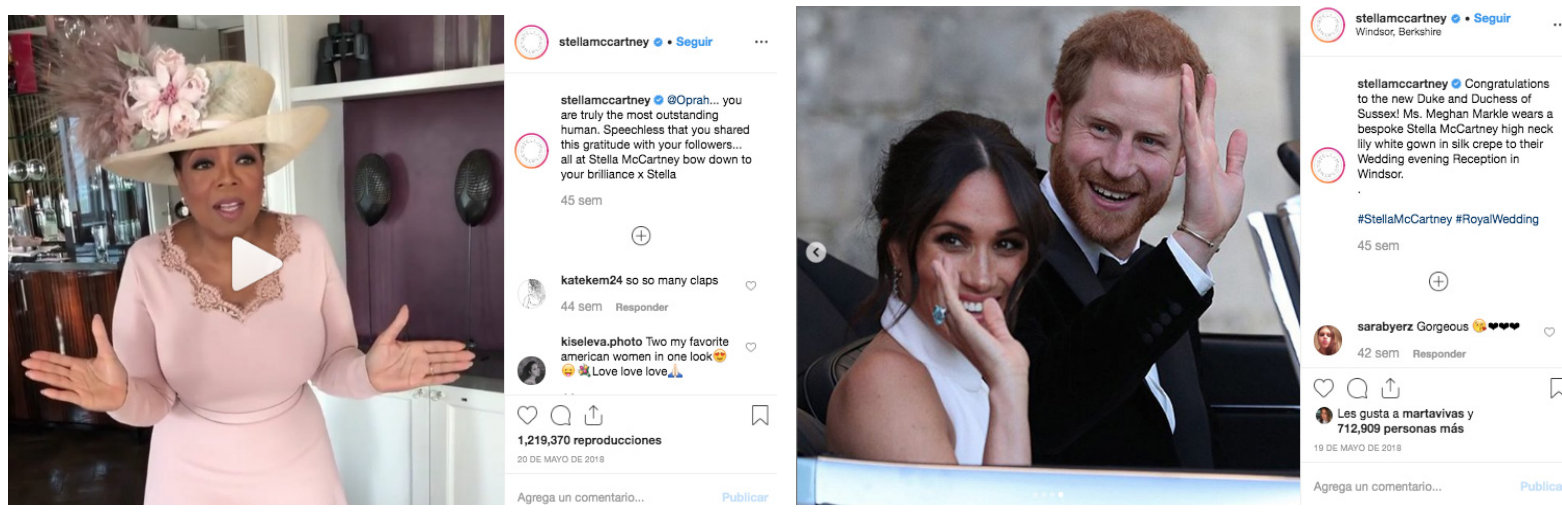

Fuente: www.instagram.com

\subsection{Actualizaciones con más engagement a lo largo del tiempo}

Finalmente, para dar respuesta a la tercera pregunta acerca de si resulta eficiente para las marcas de moda publicar contenido en los eventos especiales en los que participan, se presenta la tabla 5 con las reacciones a las publicaciones con más engagement generado a lo largo del tiempo.

Tabla 5. Estadístico descriptivo para las variables estudiadas de los posts con más engagement

\begin{tabular}{|l|l|l|l|l|l|l|l|l|l|l|}
\hline \multicolumn{2}{|l|}{} & FL & FC & FS & TL & TC & TS & \multicolumn{1}{l|}{ IL } \\
\hline \multirow{2}{*}{$\mathbf{N}$} & Válido & 10 & 10 & 10 & 10 & 10 & 10 & 10 & 10 \\
\cline { 2 - 12 } & $\begin{array}{l}\text { Perdi- } \\
\text { dos }\end{array}$ & 0 & 0 & 0 & 0 & 0 & 0 & 0 & 0 \\
\hline Media & 4914,5000 & 472,3000 & 568,6000 & 23780,0000 & 4264,1000 & 5317,7000 & 103785,4000 & 2334,3000 \\
\hline Mediana & 3918,5000 & 133,0000 & 330,5000 & 6899,0000 & 349,5000 & 796,5000 & 51121,0000 & 273,5000 \\
\hline Desviación & 5099,67653 & 782,89520 & 718,21866 & 36472,45288 & 12437,05730 & 9017,63104 & 137335,17053 & 5718,50344 \\
\hline Rango & 16319,00 & 2419,00 & 2331,00 & 116123,00 & 39646,00 & 23343,00 & 393166,00 & 18518,00 \\
\hline Mínimo & 41,00 & 2,00 &, 00 & 17,00 & 1,00 & 12,00 & 350,00 & 8,00 \\
\hline Máximo & 16360,00 & 2421,00 & 2331,00 & 116140,00 & 39647,00 & 23355,00 & 393516,00 & 18526,00 \\
\hline Total & 49145,00 & 4723,00 & 5686,00 & 237800,00 & 42641,00 & 53177,00 & 1037854,00 & 23343,00 \\
\hline
\end{tabular}

Fuente: Elaboración propia

En Facebook, la actualización que más reacciones generó logró, en total, 49.145,00 likes, lo que supone una media de 4.914,5000. Con $16.360,00$ de máximo y 41 de mínimo. Se produjeron 4.723,00 comentarios; 472,3 de media, y 5.686,00 compartidos. En Twitter se logró 237.800,00 likes, 4.2641,00 comentarios y 53177,00 retuits. Por último, 1.037.854,00 likes en Instagram y 23.343,00 comentarios.

Comparando lo anterior con los resultados obtenidos con las publicaciones emitidas a raíz de la Boda Real, se observa que los likes en Facebook son más numerosos $(68.250,00)$ que en las actualizaciones habituales $(49.145,00)$. Sin embargo, aquí los comentarios $(2.425,00)$ no son tan prolíficos como habitualmente $(4.723,00)$. En lo que respecta a los compartidos, el contenido procedente del evento especial se difunde más $(21.975,00)$ que una publicación habitual $(5.686,00)$. En lo que respecta a Twitter, los likes no son tan numerosos en la Boda $(34.934,00)$ como lo son en sus publicaciones corrientes $(237.800,00)$. Los comentarios son más limitados en el primer caso $(631,00)$ que en el segundo $(42.641,00)$; al igual que los compartidos: 9.195,00 frente a 53.177,00. En última instancia, Instagram recoge 8.892.079,00 likes en la Boda Real frente a los 
393.516,00 de las actualizaciones habituales. Se produce también un mayor número de comentarios $(43.550,00)$ en la Boda Real.

\section{Conclusiones}

Las redes sociales son ya una herramienta consolidada de relaciones públicas para transmitir mensajes entre las organizaciones y sus públicos. Su relevancia para el ámbito de la comunicación viene avalada por el número de investigaciones que centran su objeto de estudio en conocer cómo las empresas implementan este instrumento. Sin embargo, la Academia sigue aún sin poner de manifiesto cómo los públicos reaccionan a los esfuerzos comunicativos realizados por las compañías.

Frente a lo anterior, la investigación tenía por objeto dilucidar cómo las empresas internacionales del sector textil se relacionan con sus públicos en torno a un evento especial como fue la Boda Real de los Duques de Sussex. En base a esto, se formularon tres preguntas de investigación. En lo que respecta a la PI1, los resultados revelan que el esfuerzo comunicativo varía en función de la red social. Las marcas objeto de estudio son más proclives a publicar en Instagram que en Facebook o Twitter. Con la PI2 se pretendía conocer cómo responden los públicos al esfuerzo comunicativo que hacen las marcas de moda ante un evento especial. Particularmente, aunque se comparte más en Facebook que en Twitter, los usuarios generan más likes y comentarios en Instagram. Mención especial merece el escaso diálogo generado en torno al evento especial, a pesar de que la conversación es uno de los elementos fundamentales en las redes sociales. Finalmente, resulta idóneo para las marcas de moda publicar contenido relacionado con la Boda Real (PI3) sobre todo si la firma tiene presencia en Facebook o Instagram.

Analizando las reacciones de los usuarios a las actualizaciones generadas por las firmas, la presente investigación pone de manifiesto que los eventos especiales tienen más repercusión en más redes sociales, sobre todo en Facebook e Instagram; por lo que las respuestas son más numerosas cuando se participa en un evento especial. De manera general, la variable de análisis likes aumenta en tanto en cuanto el contenido responde a lo acontecido en la Boda Real. No obstante, de los resultados se infiere que se produce más diálogo en torno a las actualizaciones habituales. En lo que respecta a la naturaleza de la actualización, los posts que más repercusión alcanzaron son los que cuentan con los Duques de Sussex como protagonistas o los vestidos de novia, a excepción del caso de Dolce and Gabbana. En base a esto, la firma que logró un mayor engagement fue Givenchy y Stella McCartney, responsables de sendos vestidos de novia.

Los resultados de este estudio tienen aplicaciones prácticas dado que permiten conocer cómo se implementan los mensajes en redes sociales para poder establecer estrategias solventes en el caso de querer participar de dicho evento. Los datos dejan patente que los públicos muestran un sentimiento positivo en Facebook si los mensajes se centran en dichos actos. Al mismo tiempo, el compromiso también es mayor en Instagram dado que son más propensos a generar diálogo. En efecto, las empresas de moda alcanzan más likes en Facebook e Instagram, y sobre todo logran más conversación en Instagram si publican contenido acerca de cuál es su participación en un evento especial como es una Boda Real. También es importante tener en cuenta que el usuario muestra mayor disposición a compartir contenido en Facebook y actuar como embajador de la firma si el post responde a ese hecho particular. Es decir, es menos probable que comparta información si el contenido está relacionado con la actividad habitual de la empresa, aunque esta diferenciación no es tan marcada en Twitter. Particularmente, las firmas de moda prefieren Instagram para establecer relaciones con sus públicos. Es aquí donde más contenido se publica y donde más público es impactado. El interés por esta red social es recíproco por ambas partes dado que sus publicaciones generan más comentarios y reacciones que las actualizaciones en otras plataformas. No obstante, a la hora de compartir, y dad que no es una acción nativa de Instagram, los públicos prefieren Facebook. 
Por tanto, a pesar de que la literatura anterior ha centrado sus investigaciones en conocer el uso que las empresas le daban a las redes sociales, los resultados sugieren que debería prestarse más atención a las reacciones de los públicos. Al hilo de lo anterior, y para poder comprender cómo las empresas de moda estructuran su comunicación en torno a una Boda Real, cabe destacar que, aunque las reacciones aumentan considerablemente ante este tipo de actualizaciones, la conversación es la reacción menos generalizada. El usuario prefiere dar me gusta o compartir antes que emitir un comentario. Sin embargo, y al hilo de lo anterior, los resultados revelan una preferencia por generar conversación en Instagram por cima de las demás redes.

Concluyendo, a través del estudio se quiso conocer cuál era el esfuerzo comunicativo de una empresa de moda ante un evento especial y, más allá, conocer cómo reaccionaban los públicos ante los mensajes para finalmente argüir si resultaba beneficioso o no participar. En relación a esto, la investigación pone de relieve los beneficios en términos de engagement de participar en un acto específico como es una Boda Real y viene avalado por la medición de los tres principales indicadores con los que los públicos responden. Del mismo modo, y a pesar de que el texto se centra en el comportamiento de los públicos ante los posts de firmas internacionales a partir de una Boda Real, sería igualmente beneficioso para la disciplina de las relaciones públicas promover esta línea de investigación y estudiar el compromiso de los usuarios ante los mensajes emitidos por empresas de moda con menos proyección que participaran en otro tipo de eventos, como semanas de la moda, rompiendo así con las limitaciones que este estudio presenta. Por otro lado, responden también a futuras investigaciones aquellas llamadas a estudiar desde una perspectiva ampliamente cualitativa la naturaleza de los posts y el sentimiento de las reacciones o de los comentarios generados por los usuarios.

\section{Bibliografía}

Aaboen, L. Dubois, A. \& Lind, F. (2012). Capturing processes in longitudinal multiple case studies. Industrial Marketing Management, 41 (2), 235-246. https://doi.org/10.1016/j. indmarman.2012.01.009

Almansa Martínez, A. y Navarrete Cobo, B. (2012). Relaciones Públicas on-line: El uso de los social media en el sector hotelero de la costa del sol. En: $7^{\circ}$ Congreso Internacional de Investigación en Relaciones Públicas. Relaciones Públicas: El diálogo de las organizaciones, pp. 97-110. https://bit.ly/2qnfyog

Alonso-González, M. (2015). Social networks as communication channels of spanish fashion brands: Zara, Mango and El Corte Ingles. Index Comunicacion, 5 (1), 77-105. https://bit.ly/33adyPz

Arora, A., Bansal, S., Kandpal, C., Aswani, R. \& Dwivedi, Y. (2019). Measuring social media influencer index-insights from facebook, Twitter and Instagram. Journal of Retailing and Consumer Services, 49, 86-101. https://doi.org/10.1016/j.jretconser.2019.03.012

Barquero Cabrero, J. D. y Castillo Esparcia, A. (2011). Marco teórico y práctico de las relaciones públicas. Furtwagen.

Boyd, D. M. \& Ellison, N. B. (2008). Social network sites: definition, history, and scholarship. Journal of Computer-Mediated Communiction, 13 (1), 210-230. https://doi.org/10.1111/j.10836101.2007.00393.x

Breakenridge, D. K. (2012). Social media and public relations: Eight new practices for the PR professional. Pearson FT Press.

Carr, C. T. \& Hayes, R. A. (2015). Social Media: Defining, Developing, and Divining. Atlantic Journal of Communication, 23 (1), 46-65. https://doi.org/10.1080/15456870.2015.972282 
Castillero Ostio, E. (2014). El protocolo como gestor de públicos. Estudio sobre el ceremonial oficial en el franquismo (Tesis Doctoral). Universidad de Málaga.

Cereda, A. (2014). Modified bodies. Between fashion and identity projects. Comunicação e Sociedade, 24, 45-61. https://bit.ly/2BKaFIu

Chaplin, L. N. \& Lowrey, T. M. (2010). The development of consumer-based consumption constellations in children. Journal of Consumer Research, 36 (5), 757-777. https://doi. org/10.1086/605365

Cucinotta, C. (2017). Percursos de análise do vestuário em filmes portugueses de etnoficção. Comunicação e Sociedade, 31, 165-181. https://doi.org/10.17231/comsoc.31(2017).2610

Curtis, L., Edwards, C., Frasier, K. L., Gudelsky, S. G., Holmquist, J., Thornton, K. \& Sweetser, K. D. (2010). Adoption of social media for public relations by nonprofit organizations. Public Relations Review, 36 (1), 90-92. https://doi.org/10.1016/j.pubrev.2009.10.003

Cvijikj, I. P. \& Michahelles, F. (2013). Online engagement factors on Facebook brand pages. Social Network Analysis and Mining, 3 (4), 843-861. https://doi.org/10.1007/s13278-013-0098-8

Debatin, B., Lovejoy, J. P., Horn M.A., A.-K., Hughes, B. N. H. (2009). Facebook and online privacy: attitudes, behaviors, and unintended consequences. Journal of Computer-Mediated Communiction, 15 (1), 83-108. https://doi.org/10.1111/j.1083-6101.2009.01494.x

Dhanesh, G. S. (2017). Putting engagement in its PRoper place: State of the field, definition and model of engagement in public relations. Public Relations Review, 43 (5), 925-933. https://doi. org/10.1016/j.pubrev.2017.04.001

Diga, M. \& Kelleher, T. (2009). Social media use, perceptions of decision-making power, and public relations roles. Public Relations Review, 35 (4), 440-442. https://doi.org/10.1016/j. pubrev.2009.07.003

Esteban-Santos, L., García Medina, I., Carey, L. \& Bellido-Pérez, E. (2018). Fashion bloggers: communication tools for the fashion industry. Journal of Fashion Marketing and Management: An International Journal, 22 (3), 420-437. https://doi.org/10.1108/JFMM-10-2017-0101

Fernández Sánchez, L. F. (2004). Ceremonial y protocolo. Oberon.

Fernández Souto, A. B. (2018). Public relations and communication. Exploring opportunities in an interconnected world. Obra Digital-Revista de Comunicacion, 15, 9-12. https://bit.ly/2WIS8Gj

Fuente LaFuente, C. (2010). Protocolo oficial. Las instituciones españolas del estado y su ceremonial. Ediciones Protocolo.

Gama, M. (2014). The Ideology of Consumption in Fashion: a Diachronic Per. Comunicação e Sociedade, 24, 221-231. https://doi.org/10.17231/comsoc.24(2013).1785

García Medina, I., Miquel-Segarra, S. \& Navarro-Beltrá, M. (2018). The use of Twitter on fashion brands. A comparison between luxury and low-cost brands. Cuadernos Info, 42, 55-69. https://doi. org/10.7764/cdi.42.1349

Grunig, J. E., Hunt, T. T. (1984). Managing public relations. Holt, RinehaRT \& Winston.

Guidry, J. P.D., Jin, Y., Orr, C. A., Messner, M. \& Meganck, S. (2017). Ebola on Instagram and Twitter: How health organizations address the health crisis in their social media engagement. Public Relations Review, 43 (3), 477-486. https://doi.org/10.1016/j.pubrev.2017.04.009 
Guidry, J., Messner, M., Jin, Y. \& Medina-Messner, V. (2015). From \#mcdonaldsfail to \#dominossucks: An analysis of Instagram images about the 10 largest fast food companies. Corporate Communications: An International Journal, 20 (3), 344-359. https://doi.org/10.1108/ CCIJ-04-2014-0027

Guo, C. \& Saxton, G. D. (2014). Tweeting social change: How social media are changing nonprofit advocacy. Nonprofit and voluntary sector quarterly, 43 (1), 57-79. https://doi. org/10.1177/0899764012471585

Hernández Sampieri, R., Fernández Collado, C. y Baptista Lucío, P. (2007). Fundamentos de metodología de la investigación. McGraw-Hill.

Himelboim, I., Golan, G. J.; Moon, B. B. \& Suto, R. J. (2014). A Social Networks Approach to Public Relations on Twitter: Social Mediators and Mediated Public Relations. Journal of Public Relations Research, 26 (4), 359-379. https://doi.org/10.1080/1062726X.2014.908724

Hon, L. C. \& Grunig, J. E. (1999). Guidelines for measuring relationships in public relations. Institute for Public Relations. https://bit.ly/2NCR16R

Kemp, S. (2019). Digital 2019: Global Internet use accelerates. https://bit.ly/2WLV0BY

Kim, S., Kim, S.-Y. \& Sung, K. H. (2014). Fortune 100 companies' Facebook strategies: corporate ability versus social responsibility. Journal of Communication Management, 18(4), 343-362. https://doi.org/10.1108/JCOM-01-2012-0006

Laurell, C. (2014). Brands, community and style - exploring linking value in fashion blogging. Journal of Fashion Marketing and Management, 18(1), 3-19. https://doi.org/10.1108/JFMM-102013-0108

Laurell, C. \& Sandström, C. (2013). Value creation and appropriation in social media - the case of fashion bloggers in Sweden. International Journal of Technology Management, 61 (3-4), 309-323. https://bit.ly/2PHCNEp

Lin, S. (2018). The structural characteristics of innovation ecosystem: a fashion case. European Journal of Innovation Management, 21 (4), 620-635. https://doi.org/10.1108/EJIM-09-2017-0115

Lofman, B. (1991). Elements of Experiential Consumption: an Exploratory Study. Advances in Consumer Research, 18 (1), 729-735. https://bit.ly/33hsydV

Lovejoy, K. \& Saxton, G. D. (2012). Information, community, and action: How nonprofit organizations use social media. Journal of computer-mediated communication, 17 (3), 337-353. https://doi.org/10.1111/j.1083-6101.2012.01576.x

Machuco Rosa, A. (2013). The evolution and democratization of modern fashion: from Frederick Worth to Karl Lagerfeld's fast fashion. Comunicação e Sociedade, 24, 79-94. https://bit.ly/2NK1VYi

Mangold, W. G. \& Faulds, D. J. (2009). Social media: the new hybrid element of the promotion mix. Business Horizons, 52 (4), 357-365. https://doi.org/10.1016/j.bushor.2009.03.002

McQuarrie, E. F., Miller, J. \& Phillips, B. J. (2013). The megaphone effect: taste and audience in fashion blogging. Journal of Consumer Research, 40 (1), 136-158. https://doi.org/10.1086/669042

Miles, M. B. \& Huberman, A. M. (1994). Qualitative data analysis: An expanded sourcebook. SAGE.

Mohr, I. (2013). The impact of social media on the fashion industry. Journal of Applied Business and Economics, 15 (2), 17-22. https://bit.ly/2CeqZkR 
Nash, J. (2019). Exploring how social media platforms influence fashion consumer decisions in the UK retail sector. Journal of Fashion Marketing and Management, 23 (1), 82-103. https://doi. org/10.1108/JFMM-01-2018-0012

Oliveira, M. M. (2013). Dressing, seducing and signifying: from the symbolic dimension of fashion to the contemporary erotic imagery. Comunicação e sociedade, 24, 152-160. https://doi. org/10.17231/comsoc.24(2013).1781

Otero Alvarado, M. T. (2000). Teoría y Estructura del Ceremonial y el Protocolo. Mergablum.

Otero Alvarado, M. T. (2004). Relaciones Públicas, ceremonial y protocolo. En: Arceo Vacas, José Luis (coord.). Las Relaciones Públicas en España, pp. 277-288. McGrawHill.

Palmer, A. \& Koenig-Lewis, N. (2009). An experiential, social networkbased approach to direct marketing. Direct Mark, 3 (3), 162-176. https://doi.org/10.1108/17505930910985116

Park, J., Song, H. \& Ko, E. (2011). The Effect of the Lifestyles of Social Networking Service Users on Luxury Brand Loyalty. Journal of Global Scholars of Marketing Science, 21 (4), 182192. https://doi.org/10.1080/21639159.2011.9726521

Pérez-Curiel, C. \& Sanz-Marcos, P. (2019). Branding, influencers and new target in the communication of fashion and luxuary the Gucci tendency on Instagram. Prisma Social, 24, 1-24.

Phan, M. C., Thomas, R. \& Heine, K. (2011). Social Media and Luxury Brand Management: The Case of Burberry. Journal of Global Fashion Marketing, 2 (4), 213-222. https://doi.org/10.1080/2 0932685.2011.10593099

Piñuel, J. L. (2002). Epistemología, metodología y técnicas del análisis de contenido. Sociolinguistic Studies, 3(1), 1-42. https://doi.org/10.1558/sols.v3i1.1

Pires, H. (2014). The urban landscape and fashion advertising: the case of the DKNY brand. Comunicação e Sociedade, (26), 291-305. https://doi.org/10.17231/comsoc.26(2014).2040

Ramos Fernández, L. F. (2010). La carrera de Protocolo: Controversia crítica del nuevo grado desde los Estudios de Relaciones Públicas". En: $6^{\circ}$ Congreso Internacional de Investigación y Relaciones Públicas, pp. 79-95. https://bit.ly/2NCU6ni

Richter, D., Riemer, K. y Vom Brocke, J. (2011). Internet social networking: research state of the art and implications for Enterprise 2.0 (state of the art). Business \& Information System Engineering, 53 (2), 89-103. https://doi.org/10.1007/s12599-011-0151-y

Ruehl, C. \& Ingenhoff, D. (2015). Communication management on social networking sites Stakeholder motives and usage types of corporate Facebook, Twitter and YouTube pages. Journal of Communication Management, 19 (3), 288-302. https://doi.org/10.1108/JCOM-04-2015-0025

Rybalko, S. \& Seltzer, T. (2010). Dialogic communication in 140 characters or less: How Fortune 500 companies engage stakeholders using Twitter. Public Relations Review, 36 (4), 336-341. https://doi.org/10.1016/j.pubrev.2010.08.004

Sánchez González, D. M. (2011). Fundamentos del ceremonial y el protocolo. Síntesis.

Sánchez González, D. M., Gómez Requejo, M. y Pérez Marcos, R. M. (2015). Historia del ceremonial y del protocolo. Síntesis.

Saxton, G. D. \& Waters, R. (2014). What do stakeholders like on Facebook? Examining public reactions to nonprofit organizations' informational, promotional, and community- 
building messages. Journal of Public Relations Research, 26 (3), 280-299. https://ssrn.com/ abstract $=2536982$

Sullivan, S. J., Schneiders, A. G., Cheang, C.-W., Kitto, E., Lee, H., Redhead, J., Ward, S., Ahmed, O. H. \& McCrory, P. R. (2012). 'What's happening?'A content analysis of concussionrelated traffic on Twitter. British Journal of Sports Medicine, 46 (4), 258-263. http://dx.doi. org/10.1136/bjsm.2010.080341

USC Annenberg Center for Public Relations (2019). PR:TECH. The future of technology in communication. https://bit.ly/34zsA0K

Verhoeven, P., Tench, R., Zerfass, A., Moreno, Á. \& Vercic, D. (2012). How European PR practitioners handle digital and social media. Public Relations Review, 38 (1), 162-164. https:// doi.org/10.1016/j.pubrev.2011.08.015

Vieira, V. A. (2009). An extended theoretical model of fashion clothing involvement. Journal of Fashion Marketing and Management, 13 (2), 179-200. https://doi. org/0.1108/13612020910957707

Waters, R. D., Burnett, E., Lamm, A. \& Lucas, J. (2009). Engaging stakeholders through social networking: How nonprofit organizations are using Facebook. Public Relations Review, 35 (2), 102-106. https://doi.org/10.1016/j.pubrev.2009.01.006

Waters, R. D. \& Jamal, J. Y. (2011). Tweet, tweet, tweet: A content analysis of nonprofit organizations' Twitter updates. Public Relations Review, 37 (3), 321-324. http://dx.doi. org/10.1016/j.pubrev.2011.03.002

Wright, D. K. \& Hinson, M. D. (2013). An updated examination of social and emerging media use in public relations practice: A longitudinal analysis between 2006 and 2014. Public Relations Journal, 8 (2), 1-39. http://dx.doi.org/10.1016/j.pubrev.2011.03.002

Yin, R. K. (1994). Investigación sobre estudio de casos. Diseño y métodos. Applied Social Research Methods Series, 5 (2), 1-35. https://bit.ly/2qAr4Nt 